\title{
Biofunctional Micropatterning of Thermoformed 3D Substrates
}

\author{
Björn Waterkotte, Florence Bally, Pavel M. Nikolov, Ansgar Waldbaur, Bastian E. Rapp, \\ Roman Truckenmüller, Jörg Lahann, Katja Schmitz,* and Stefan Giselbrecht*
}

Mimicking the in vivo microenvironment of cells is a challenging task in engineering in vitro cell models. Surface functionalization is one of the key components providing biochemical cues to regulate the interaction between cells and their substrate. In this study, two different approaches yield biofunctional surface patterns on thermoformed polymer films. The first strategy based on maskless projection lithography enables the creation of grayscale patterns of biological ligands with a resolution of $7.5 \mu \mathrm{m}$ in different shapes on a protein layer adsorbed on a polymer film. In the second strategy, polymer films are micropatterned with different functional groups via chemical vapor deposition polymerization. After thermoforming, both types of pattern can be decorated with proteins either by affinity binding or covalent coupling. The 3D microstructures retain the biofunctional patterns as demonstrated by selective cell adhesion and growth of L929 mouse fibroblasts. This combination of functional micropatterning and thermoforming offers new perspectives for the design of 3D cell culture platforms.

\section{Introduction}

Microstructured surfaces play an important role in studying cellular behavior in response to biochemical and biophysical signals. Substrates that facilitate site-specific activation of cell signaling via controlled immobilization of bioactive molecules are necessary for mimicking the intricate extracellular environment of cells in vitro. ${ }^{[1]}$ Tailor-made arrangement of biomolecules on surfaces can be used to precisely guide cell behavior or control (stem) cell fate in defined artificial environments for future applications. To obtain biofunctional surfaces, amongst others the mode of immobilization, the distribution of the tethered molecules on a micrometer scale and the microtopography of the substrate need to be tailored. ${ }^{[2]}$ So far, in vitro studies were mainly carried out on planar surfaces. To permit highly miniaturized and thus parallelized assays with low compound consumption, for instance to test the response of cells to effector molecules, microarrays of protein or ligandcoated spots ranging from $100-500 \mu \mathrm{m}$ in diameter can be produced by microcontact printing, spotting or patterning with microfluidic networks. ${ }^{[3]}$

To investigate the role of surface bound chemical cues on cell behavior, often patterns of biomolecules, particularly proteins, such as growth factors or cell adhesion proteins, have to be created. Biologically active molecules can be immobilized on chemically modified patterns on the substrate. As in the production of microarrays, spatially-defined patterns of functional groups have been generated by microcontact printing ${ }^{[4]}$ and microfluidic networks, ${ }^{[5]}$ but they can also be drawn by dip pen nanolithography (DPN) using the tip of a probe controlled by an atomic force microscope, ${ }^{[6]}$ or created by mask-based lithography with biocompatible resists. ${ }^{[7]}$ Another option to obtain patterns of functional groups is chemical vapor deposition (CVD) polymerization of [2.2]paracyclophane derivatives. ${ }^{[8]}$ This

\author{
B. Waterkotte, Prof. K. Schmitz \\ Institute of Organic Chemistry (IOC) \\ Karlsruhe Institute of Technology (KIT) \\ Fritz-Haber-Weg 6, Karlsruhe, 76131, Germany \\ E-mail:Schmitz@biochemie-TUD.de \\ B. Waterkotte, Dr. F. Bally, Prof. J. Lahann, Prof. K. Schmitz \\ Institute of Functional Interfaces (IFG) \\ Karlsruhe Institute of Technology (KIT) \\ Hermann-von-Helmholtz-Platz 1, Eggenstein-Leopoldshafen \\ 76344, Germany \\ P. M. Nikolov, Dr. S. Giselbrecht \\ Institute for Biological Interfaces (IBG1) \\ Karlsruhe Institute of Technology (KIT) \\ Hermann-von-Helmholtz-Platz 1, Eggenstein-Leopoldshafen \\ 76344, Germany \\ E-Mail: Stefan.giselbrecht@kit.edu
}

DOI: 10.1002/adfm201301093

\author{
A. Waldbaur, Dr. B. E. Rapp \\ Institute of Microstructure Technology (IMT) \\ Karlsruhe Institute of Technology (KIT) \\ Hermann-von-Helmholtz-Platz 1 \\ Eggenstein-Leopoldshafen, 76344, Germany \\ Dr. R. Truckenmüller \\ MIRA Institute for Biomedical Technology \\ and Technical Medicine \\ University of Twente \\ Drienerlolaan 5, Enschede, 7522 NB, The Netherlands \\ Prof. J. Lahann \\ Biointerfaces Institute \\ University of Michigan \\ 2800 Plymouth Rd., Ann Arbor/MI, 48109, USA \\ Prof. K. Schmitz \\ Clemens-Schöpf-Institute of Organic Chemistry and Biochemistry \\ Technical University of Darmstadt \\ Petersenstraße 22, Darmstadt, 64287, Germany.
}


solvent-free process enables the fabrication of homogeneous, pinhole-free thin polymer coatings on any substrate material. Depending on the precursor used for CVD (co)polymerization, the desired functional groups are provided by the reactive polymer coating. Patterning is possible either during the CVD process, for instance by vapor-assisted micropatterning in replica structures (VAMPIR), ${ }^{[9]}$ or after CVD polymerization. ${ }^{[10]}$ Post-CVD modification of the substrate resembles commonly used techniques for protein patterning as described above. For instance, microcontact printing is frequently applied to create micropatterns of proteins or linkers for the attachment of bioactive molecules. ${ }^{[4,11]}$ While this technique, along with other methods based on masks or stamps leads to binary patterns, different protein densities can be obtained by microfluidic technologies ${ }^{[5]}$ or by photolithography in combination with photoreactive groups or photocleavable protecting groups. The technique of protein adsorption by photobleaching (PAP), introduced by Holden and Cremer, ${ }^{[12]}$ uses fluorophors as photoreactive groups that covalently interact with the surface and can be applied to tether ligands for protein immobilization to the surface by the use of fluorophores conjugated with the respective ligand or functionality. ${ }^{[13]}$ By using a photomask, ${ }^{[12]}$ a dynamic crystal display, ${ }^{[13]}$ laser writing ${ }^{[14]}$ or dynamic mirror displays, ${ }^{[15]}$ ligand patterns can be generated by PAP. The amount of immobilized ligand correlates with the dose of irradiation light so that "grayscale patterns" of ligands (and thereby proteins) can be accomplished. In particular, the combination of maskless projection lithography (MPL) with PAP as a patterning strategy (MPL-PAP) allows for high flexibility in the created patterns. ${ }^{[15]}$

However, the rigid planar surfaces generally used for microcontact printing, CVD and lithography are far from the 3D display of diverse ligands, to which cells respond in their native environment of adjacent cells within a tissue, ${ }^{[16]}$ and cells have been found to react highly sensitively to the spatial distribution of immobilized peptides and proteins. ${ }^{[17]}$ Therefore, the fabrication of 3D functionalized substrates is crucial for mimicking cell environment. ${ }^{[18]}$ To engineer 3D cell culture platforms, different geometries with various size ranges, such as microwells with 50-300 $\mu \mathrm{m}$ in diameter for single cell experiments or channels with a width of $5-50 \mu \mathrm{m}$ to mimic lymph or blood capillaries, have been used. ${ }^{[19]}$ Microtechniques, to create such structures, have been developed for high-throughput screening in drug discovery and biotechnology. ${ }^{[20]}$ Such microfluidic systems permit an improved spatiotemporal resolution, which enables precise manipulation of fluids, particles, cells or microtissues on a micrometer scale.

However, conventional microtechniques typically enable the fabrication of devices with homogeneous chemical and physical properties, while the patterning techniques described above are common to functionalize planar substrates but mostly inapplicable for non-planar structures since vertical sidewalls or undercut regions remain almost inaccessible for controlled patterning. Creating patterns of biomolecules such as proteins, DNA or oligosaccharides on planar polymer substrates to be mechanically structured in a later step appears to be a solution. However it bears the risk of inactivation and degradation of these sensitive compounds for example under elevated temperatures and the mechanical stress applied for fabrication of 3D structures.
A new process to create patterned 3D structures on the micro- and even nanometer scale was developed in the labs of Truckenmüller and Giselbrecht, who showed that a prepatterning of thin polymer films can be preserved in a solidstate forming step, referred to as microscale thermoforming or microthermoforming. ${ }^{[21]}$ In this process, the polymer films are heated to a softened state and formed to thin-walled microdevices by $3 \mathrm{D}$ stretching. ${ }^{[22]}$ As the pre-patterning process is carried out on the initially planar polymer films, polymer modification techniques, allowing for a high spatial resolution, can be applied. After thermoforming of the film in a softened state, which allows maintenance of the modifications due to the permanent material cohesion of the film, the pre-patterned filmbased devices can be further modified in so-called post-process step. The three-step SMART approach (Substrate Modification and replication by thermoforming) combines microthermoforming with various material modification techniques such as nanoimprinting or lithography to create film-based microwell arrays with overlaid micro- and nanoscale topographies or cell adhesion patterns even on the vertical sidewalls of the wells. ${ }^{[21 a, 23]}$ However, the creation of patterned biofunctional 3D surfaces by a site-specific coupling of sensitive bioactive molecules, such as proteins, within corresponding microstructures remains a challenge. ${ }^{[24]}$

In this paper, we present a strategy to overcome this limitation by splitting up the functionalization procedure in a pre-patterning process of heat-stable linkers or moieties prior to forming and the conjugation of heat-sensitive molecules to these pre-defined sites after forming in the post-process in order to design microdevices with $3 \mathrm{D}$ patterned biofunctionalized surfaces. Spatially-defined patterns of chemical functionalities and small ligand molecules of enhanced thermostability have been created by CVD polymerization and MPLPAP, respectively, on planar polymer film substrates, which have subsequently been thermoformed and finally decorated with sensitive molecules, in this case proteins. We focused on investigating the combination of CVD and MPL-PAP with microthermoforming as a new versatile platform that enables fast and convenient generation of biomolecular patterns within film-based 3D microstructures.

\section{Results}

\subsection{General Routes to Create Biofunctional Surface Patterns on Thermoformed Substrates}

To create functional patterns on thermoformed polymer films, we used two different routes: a lithography-based and a CVDbased approach. To illustrate the overall workflow and the differences of the patterning strategies, the two routes are depicted in Figure 1.

The initial step is functional patterning of the polymer substrate either with MPL-PAP (Figure 1, 1-4) or CVD polymerization (Figure 1, 7-10). For MPL patterning a digital-mirror-based set-up creates a patterned light beam that is projected onto the sample (Figure 1, 3). The protein coated surface (Figure 1, 2) is immersed in a biotinylated-fluorophore solution. Upon irradiation, a primary pattern of immobilized biotin-fluorescein is 
maskless lithography

(1)

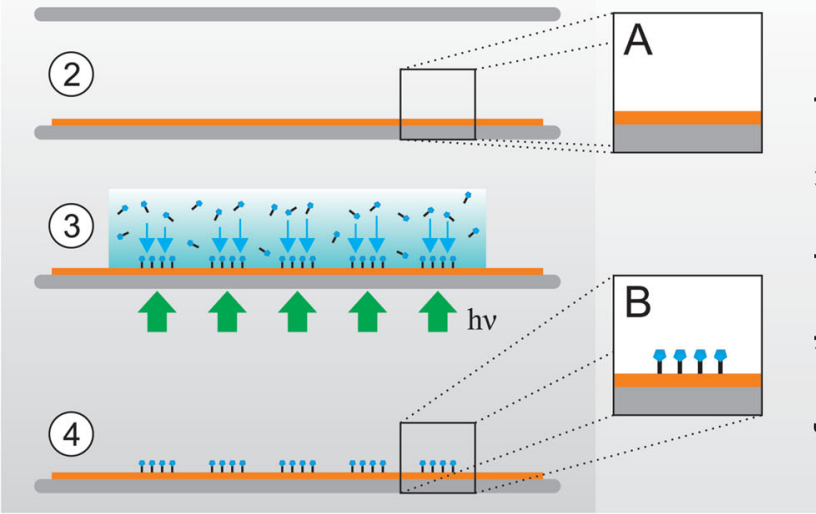

\section{chemical vapor deposition}

(7)

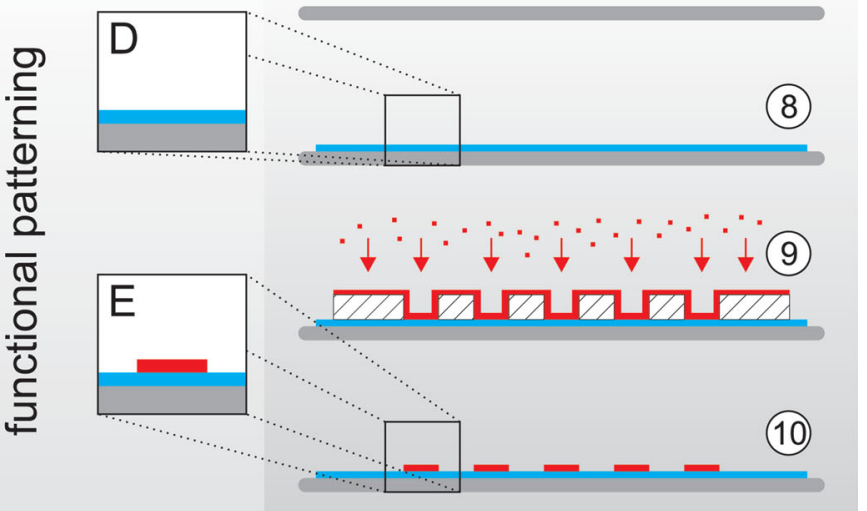

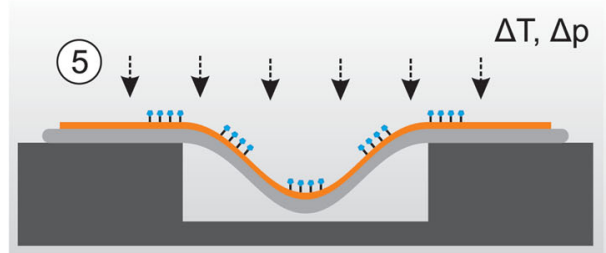

$\Delta \mathrm{T}, \Delta \mathrm{p}$

thermoforming

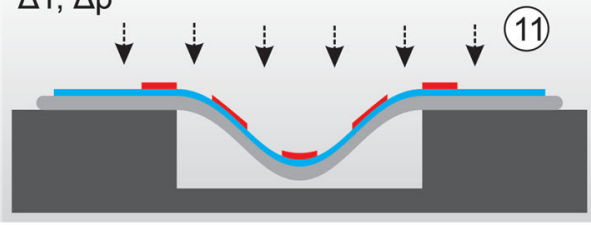

(6)
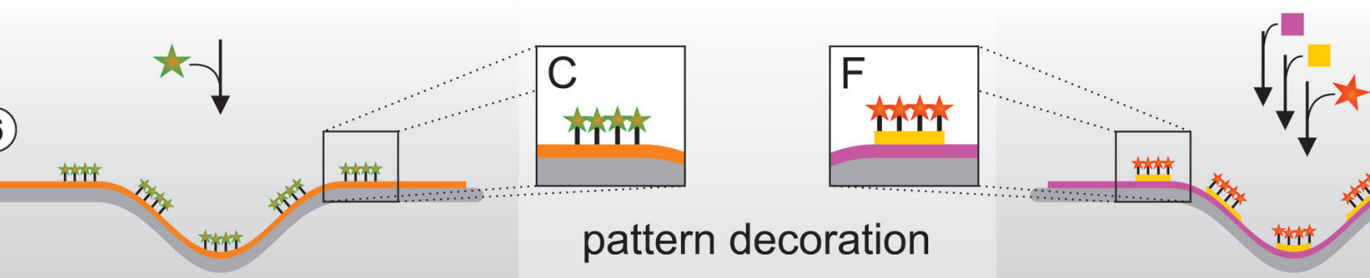

Figure 1. Combination of functional patterning with thermoforming. In the maskless projection lithography (MPL) approach, the thin polymer film (1) is initially coated by adsorption of a protein (2) such as BSA or fibronectin (2, A). This surface is then structured by MPL-PAP using a fluorescein-biotin conjugate (3) which creates a pattern of immobilized biotin on the protein layer (4, B). The sample is thermoformed (5) and the pattern is decorated with streptavidin-Cy3 for visualization (6, C). For the chemical vapor deposition (CVD) route, the thin polymer film (7) is initially CVD-coated (8) with poly(p-xylylene-4-methyl-2-bromoisobutyrate-co-p-xylylene) (8, D). A PDMS stencil (hatched blocks) is applied to the surface and a second CVD step (9) then covers unprotected areas with poly(p-xylylene-4-carboxylic acid pentafluorophenolester-co-p-xylylene). This pattern (10, E) is thermoformed (11) and coated with cell-repellant poly[poly(ethylene glycol) methyl ether methacrylate] in the bromoisobutyrate-functionalized areas and with the desired molecule, fibronectin or biotin, in the areas coated with pentafluorophenolester groups, subsequently stained with streptavidin-Cy3 (12, F).

created (Figure 1, 4, B). The surface density of biotin molecules is proportional to the intensity of the incident light (see Supporting Information, Figure S4). The projection of grayscale patterns with the DMD allows generation of arbitrary grayscale distribution of molecules on adsorbed protein films. ${ }^{[15]}$

For the CVD polymerization approach, two successive deposition steps are performed on the films to generate a pattern of two functional polymer layers via VAMPIR. ${ }^{[9]}$ First, the sample is homogeneously coated with a 2-bromoisobutyrate-functionalized xylylene copolymer, acting as an initiator for later ATRP step. Then, a PDMS stencil is sealed to the surface (Figure 1, 9) to prevent further deposition on some areas of the substrate. The second CVD step creates the functional pattern by polymerization of an active ester, the poly( $p$-xylylene-4-carboxylic acid pentafluorophenolester-co- $p$-xylylene) on the surface as a negative of the PDMS stencil microstructure (a pattern of squares is created) (Figure 1, 10). Via CVD polymerization, a wide range of defined chemical functionalities can be anchored on the surface ${ }^{[25]}$ and derivatized during subsequent steps. The films harboring the primary pattern created by either of the two strategies are then formed in their entropy-elastic state. ${ }^{[22]}$ Afterwards, the 3D film-based microstructures are decorated. Bioactive micropatterned surfaces are thus applicable to 3D cell culture platforms.

\subsection{Biofunctionalization of Patterned Planar Substrates and Cell Cultures}

The resolution of the MPL-PAP device is about $2.5 \mu \mathrm{m}$ per pixel for flat and even surfaces such as glass. ${ }^{[15]}$ This resolution could be reproduced for individual thin polymer film samples (see Supporting Information, Figure S4). However, due to variations in substrate height and the sensitivity of projection lithography to small variations in working distance, the typical resolution on polymer films was found to be $7.5 \mu \mathrm{m}$ before and after forming 
A)

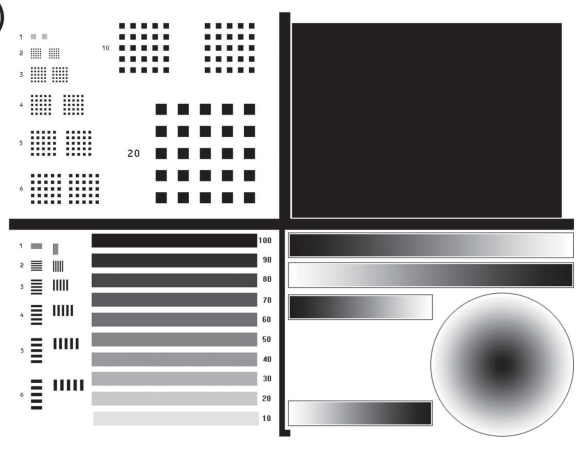

B)

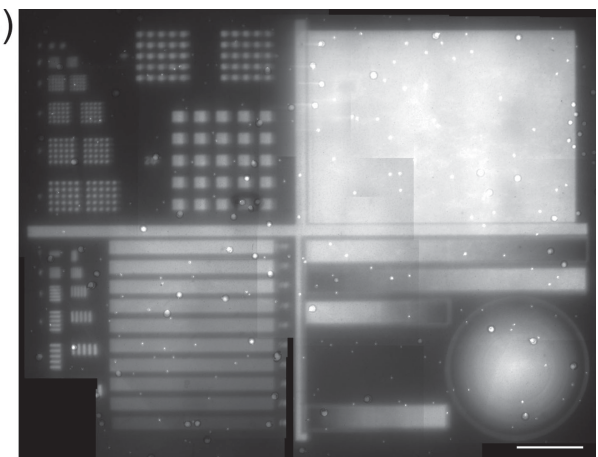

Figure 2. Digital original and COP film with protein pattern created by MPL-PAP. A) Digital 8 bit grayscale image that was projected. The negative of the depicted image was illuminated. B) Fluorescence microscopy image of streptavidin-Cy3 stained pattern. The image consists of 21 individual images $(1.4 \mathrm{~mm} \times 1 \mathrm{~mm})$, stitched with FIJI. Scale bar: $500 \mu \mathrm{m}$.

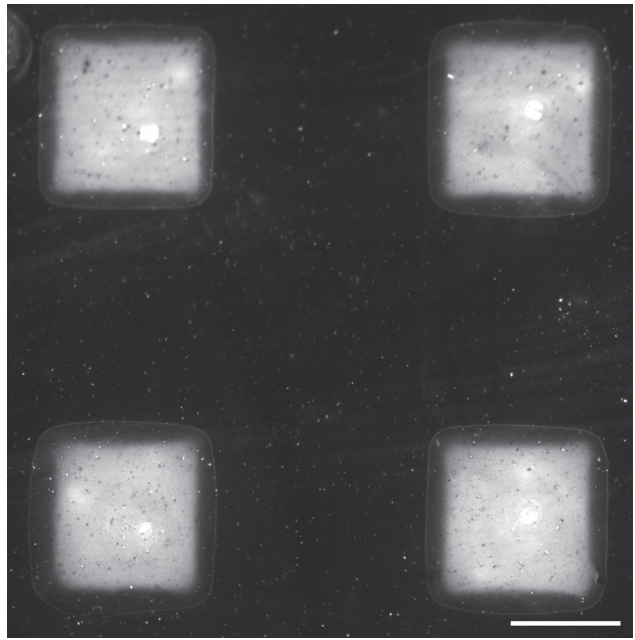

Figure 3. Fluorescent image of CVD pattern on flat COP film. Squares are decorated with biotin and stained with streptavidin-Cy3. Scale bar: $200 \mu \mathrm{m}$ microscopy reveals the presence of biotin exclusively on the squares of the pattern (in this case $250 \mu \mathrm{m} \times 250 \mu \mathrm{m}$ edge length).

The biocompatibility of the functionalized surfaces was then tested by L929 mouse fibroblast cultivation on the patterns under standard conditions. ${ }^{[28]}$ With MPL-PAP, a pattern was generated on a fibronectin coated COP film and decorated with streptavidin-Cy3, prior to cell seeding. Streptavidin-Cy3 was applied as a placeholder to visualize the pattern and was not expected to influence cells. Using streptavidin-conjugated biomolecules for future studies will therefore allow for investigation of the impact of patterned effector molecules on cell behavior without affecting cell attachment. After $72 \mathrm{~h}$ of incubation, fibroblasts showed normal growth without any detrimental changes in morphology pointing to stress, such as membrane blebbing or shrinkage (Figure 4A).

The live-dead staining based on the membrane permeability of PI and enzymatic processing of Calcein-AM is frequently applied to estimate cell viability. ${ }^{[29]}$ Upon uptake, Calcein-AM is modified into membrane-impermeable calcein, so that only viable cells retain it in the cytoplasm where chelatization of (see Supporting Information, Figures S4, S5). The digital master and a fluorescently-stained microstructure on a COP planar surface are depicted in Figure 2. The bright spots distributed all over the image come from surface defects on the thin polymer films, observed on all COP substrates.

Alternatively, primary patterns of reactive coatings have been created on COP films by CVD polymerization, applying the VAMPIR process (Figure 3).

The background of the pattern, bearing the ATRP initiator, was PEGylated by ATRP of poly(ethylene glycol) methyl ether methacrylate (PEGMA). ${ }^{[26]}$ Amine-functionalized biotin was subsequently immobilized by active ester-amine coupling ${ }^{[27]}$ on the squares of the pattern. After conjugation of fluorescently-labeled streptavidin, fluorescence
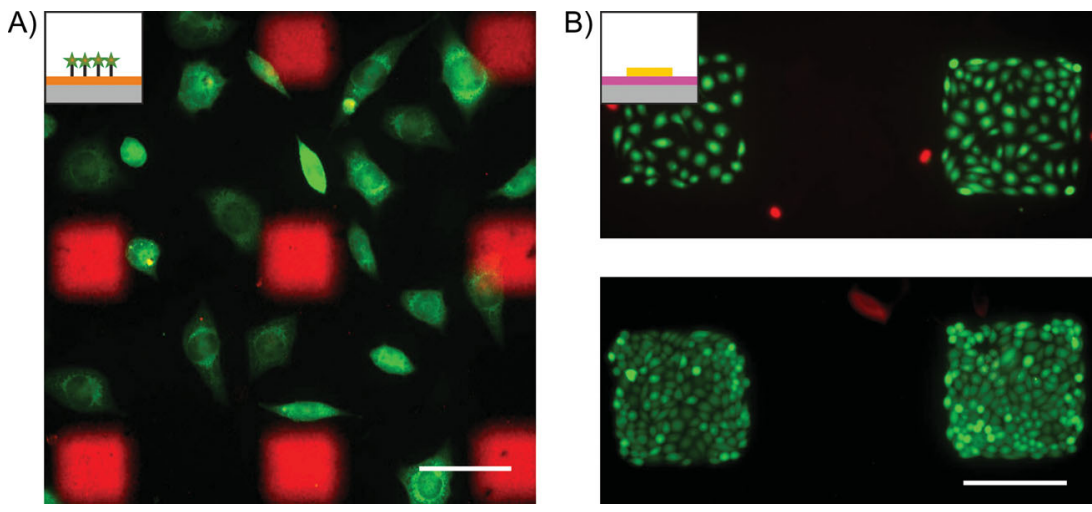

Figure 4. Biocompatibility test of functionalized surfaces with L929 mouse fibroblasts on COP films. A) Cell adhesion on non-responsive patterns created by MPL-PAP on a fibronectin coating. The squares were visualized with streptavidin-Cy3 (red). Scale bar: $100 \mu \mathrm{m}$. B)Controlled cell adhesion on fibronectin-decorated squares, created by CVD patterning. The background was passivated by PEGylation. In both cases, cytoplasm of viable cells was stained green by CalceinAM and dead cells were stained with Propidium iodide (red). Scale bar: $300 \mu \mathrm{m}$. 
A)

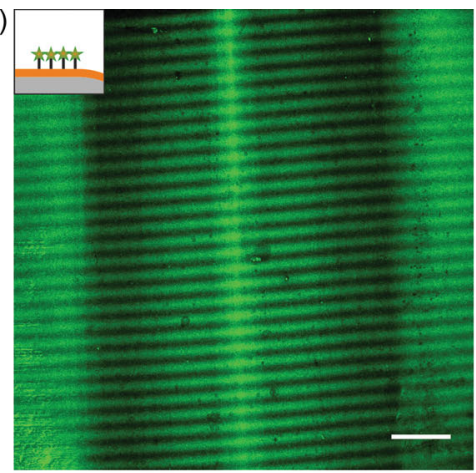

B)

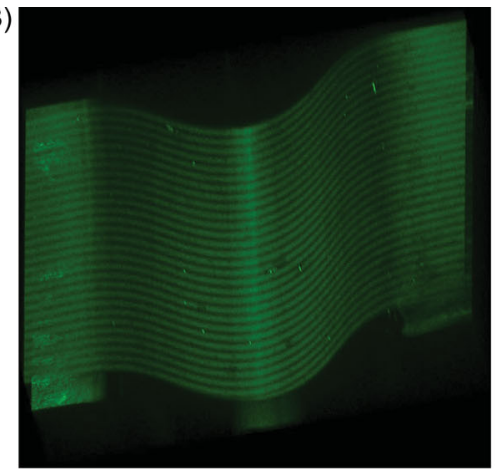

$\underset{\rightarrow}{\text { B.1 }}$

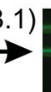

Figure 5. Confocal fluorescent images of thermoformed COP film with a streptavidin-Alexa488 stained protein pattern created by MPL-PAP. A) Z-projection of the line pattern, line width and distance $25 \mu \mathrm{m}$. Scale bar: $200 \mu \mathrm{m}$. B) 3D reconstruction of formed channel. Area shown is $1550 \mu \mathrm{m} \times 1550 \mu \mathrm{m}$. B.1) Y-projection of the channel. Arrows indicate the areas with increased signal intensity, due to scattering and reflection from the back of the film. Scale bar: $200 \mu \mathrm{m}$.

intracellular calcium leads to green fluorescence of the molecule. PI only penetrates damaged cell membranes and intercalates between base pairs of double stranded DNA in the nucleus leading to a red stain of the nuclei. ${ }^{[30]}$ In our experiments the combination of Calcein-AM and PI staining revealed that cells were viable on the MPL-PAP-patterned surfaces.

Surface patterns created by CVD polymerization were chemically derivatized to restrict cell growth to a defined square pattern. The film surface with bromoisobutyrate functionalization was selectively PEGylated by ATRP of PEGMA for low protein adsorption, ${ }^{[26,31]}$ and fibronectin was immobilized on squares of the pattern by active ester-amine coupling. Live-dead staining with Calcein-AM and $\mathrm{PI}^{[23 \mathrm{~b}, 32]}$ demonstrated that the highest number of viable cells adhered on the fibronectin squares. Only very few dead cells were detected especially on the edges of the square patterns. Fibroblasts growth was restricted to the

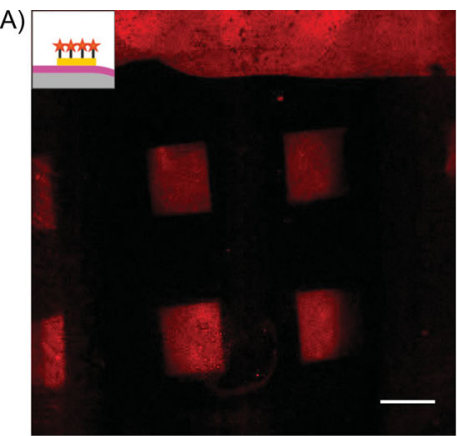

B)

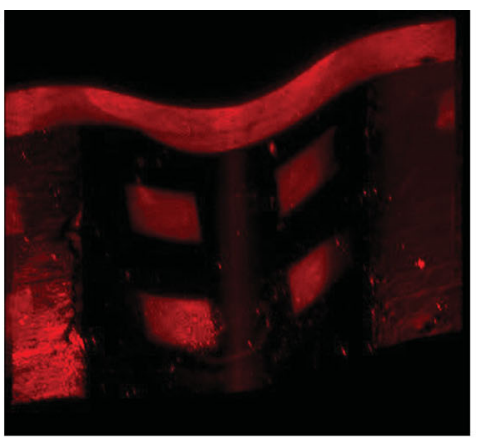

$\rightarrow$

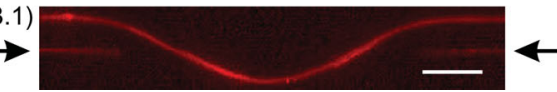

Figure 6. Confocal fluorescence images of thermoformed COP film with streptavidin-Cy3 stained biotin pattern created by CVD. A) Z-projection of the square pattern. Scale bar: $200 \mu \mathrm{m}$. B) 3D reconstruction of formed channel. Area shown is $1550 \mu \mathrm{m} \times 1550 \mu \mathrm{m}$. B.1) Y-projection of the channel. Arrows indicate the areas with increased signal intensity, due to scattering and reflection from the back of the film. Scale bar: $200 \mu \mathrm{m}$. protein-coated squares while the PEGylated areas remained nearly cell-free as expected (Figure 4.B).

\subsection{Functional Micropatterns after Thermoforming}

COP films that were patterned using the MPL-PAP or CVD approach were subsequently thermoformed to create $3 \mathrm{D}$ substrates. As a model shape that is relevant e.g. in cardiovascular studies, a channel structure was formed with a surface featuring planar primary patterns of different functionalities. The forming conditions of $140{ }^{\circ} \mathrm{C}$ and $1 \mathrm{MPa}$ were applied to form rounded channels with a depth of $318 \mu \mathrm{m} \pm 8 \mu \mathrm{m}$ and an average curvature radius of $394 \mu \mathrm{m} \pm 2 \mu \mathrm{m}$, although a rectangular-shaped negative mould cavity was used. The curvature radius of thermoformed structures can easily be controlled by choosing different forming temperatures, gas pressures or ratios of film thickness to mould cavity dimensions such that the polymer film is free-formed and not completely forced against all mould cavity walls. The presented microchannel design led to a maximum local areal draw ratio at the bottom of the channel of about 1:1.1. To compensate possible higher local areal draw ratios, the pattern could be adapted by pre-distortion, as commonly applied in macroscopic thermoforming of packaging, to compensate the effect of 3D stretching.

To demonstrate the feature preservation after thermoforming, a periodic line pattern of immobilized biotin molecules, obtained by MPL-PAP, was formed and then incubated with fluorescently-labeled streptavidin for visualization. The microstructure created by MPL-PAP was stable during thermoforming since the primary biotin line pattern had been preserved throughout the forming process. It could be decorated with streptavidin-Cy3 after thermoforming and detected under a fluorescence microscope (Figure 5A and B). The bright areas at the right and left side of the projection (Figure 5.A) derive from reflections from the back of the COP film as depicted in Figure 5B.1 (black arrows).

COP films coated with a patterned reactive polymer via CVD were also shaped by thermoforming. Afterwards, the background was PEGylated and the squares were decorated with biotin and stained with fluorescentlylabeled streptavidin. Patterns within the thermoformed channel clearly followed the curvature of the structured surface as demonstrated by a 3D reconstruction of distinct images (Figure 6).

The homogeneously stained line at the top of the image shows an area where the patterning mask ended. The slightly irregular transition line derives from manual 


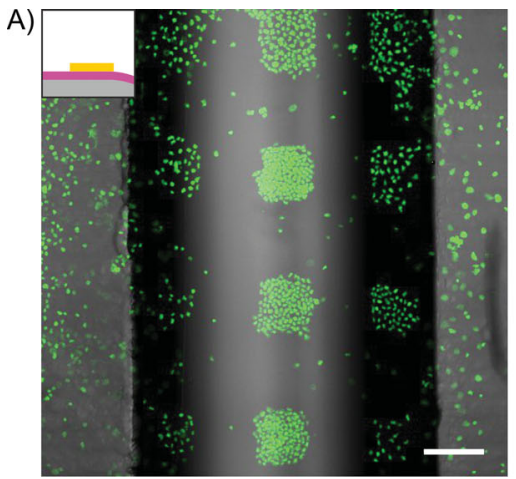

B)

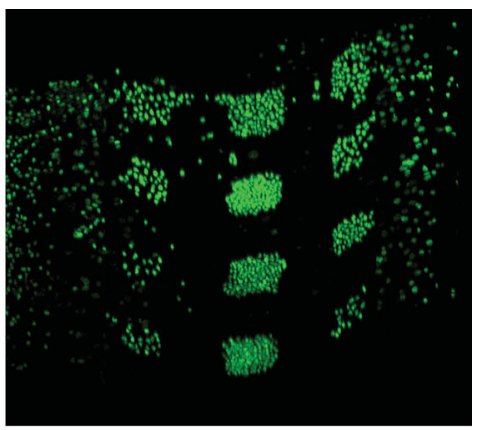

B.1)

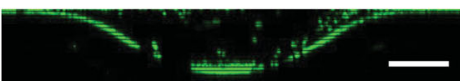

Figure 7. L929 fibroblasts in a CVD-patterned channel. Squares were functionalized with fibronectin, whereas the remaining surface was passivated by PEG. The live-dead staining with Syto 16 (green) and Propidium iodide (red) is shown. A) Overlay of Z-projections of cell fluorescent and brightfield images (gray) of the channel. B) 3D reconstruction of the cells, growing on patterns within the channel. B.1) Y-projection of the channel. Scale bar: $200 \mu \mathrm{m}$.

cutting of the PDMS mask sealed to create the square pattern (Figure 1, 9). The bright areas at the left and right sides of the projection in Figure 6B come from reflections from the back of the film as depicted in Figure 6 B.1 (black arrows).

The effect of a biofunctional micropattern based on a PEGylated background and fibronectin immobilization on cell growth was also demonstrated in a thermoformed channel (Figure 7).

The bioactive pattern led to a distinct organization of adherent cells on the squares within the channel. However, the squares of the pattern at the sidewalls of the channel harbored slightly fewer cells than the squares at the bottom which was a consequence of our simplified seeding protocol. Cells were seeded in three sequential steps while the channel was stepwise rotated by approximately $0^{\circ},+60^{\circ}$ and $-60^{\circ}$ around its long axis to enable a potentially homogenous settlement of cells to the bottom and also to the sidewalls. In Figure 7.A, an overlay of the brightfield image with the fluorescent images illustrates the selective cell adhesion on the fibronectin-functionalized areas within the microchannel.

\section{Discussion}

Many studies have demonstrated that various signals from the cell environment, including curvature radii, ${ }^{[33]}$ substrate stiffness, ${ }^{[34]}$ topographies, ${ }^{[35]}$ and biochemical surface properties ${ }^{[36]}$ play a crucial role for influencing cell morphology, adhesion, migration, proliferation, apoptosis, differentiation and senescence. Meanwhile, there are many sophisticated methods available for generating patterned biofunctionalized planar surfaces. The engineering of surface patterns on vertical sidewalls or curvilinear surfaces of more complex technical 3D environments, such as microwell arrays or microfluidic channels, is still limited due to low accessibility of their surfaces. Therefore the most efforts have been focused on mimicking only a single or a combination of very few microenvironmental components in 3D microdevices for example by combining homogeneous surface coatings of $3 \mathrm{D}$ surfaces of microwells or by patterning of the bottom of microchannels. ${ }^{[17 b, 37]}$

In contrast, chemical patterning on 3D surfaces was rarely approached. One early strategy to create a pattern on curved substrates was the transfer from a planar PDMS stamp to a curved surface by microcontact printing. ${ }^{[38]}$ The approach was used to create submicron patterns on convex substrates with radii of curvature of $25 \mu \mathrm{m}$ but is not applicable to concave structures. Inverted microcontact printing was also applied to create functionalized areas on 3D polymer surfaces. ${ }^{[39]}$ Typically the surface and the inner cavity surface are differently functionalized. Biochemical patterning within cavities has been not demonstrated with this approach. Chen and Lahann introduced a mask-based photopatterning approach to create square patterns within a microchannel with a depth of $50 \mu \mathrm{m} \cdot{ }^{[10 \mathrm{~b}]}$ The pattern was successfully transferred to the substrate, but the mask-based approach led to blurred shapes at the bottom of the channel. In addition, the channel walls were hardly accessible for controlled patterning by this strategy. Lithography-based techniques to create 3D cell adhesion patterns in microstructures based on the SMART approach have also been shown in the past. ${ }^{[21 a, 23 b]}$ For instance, a mask-based patterning by deep UV-irradiation of polystyrene was applied to pattern cell adhesion on the curvilinear surfaces within microcavities. However, these methods did not allow an advanced control of chemical surface functionalization. Furthermore, microfabrication processes combined with intricate surface chemistry protocols has typically led to establishment of elaborate multistep protocols, which prevent the up-scaling of related in vitro assays and their statistical validation.

In this work, we demonstrated that combination of thermoforming with MPL-PAP or CVD serves as simple and robust technical platform for fabrication of 3D biofunctionalized patterned microstructures. The key of our approach is the generation of a primary chemical pattern that is stable during the thermoforming process and subsequently decorated. This procedure allows a highly controlled patterning with a high lateral resolution on a flat substrate, without affecting or limiting the following thermoforming procedure and at the same time circumventing the challenges of directly patterning on a three dimensional substrate.

The PAP approach applied in combination with MPL allows for a straightforward integration into existing standard in vitro cell culturing experiments based on fibronectin or other physisorbed proteins. ${ }^{[40]}$ With MPL-PAP, a wide range of surface function densities can be deposited such as linear or circular gradients or any other arbitrary pattern. Moreover, the type of pattern can be freely chosen for every exposition, thus a wealth of different patterns is easily accessible. With a resolution of $7.5 \mu \mathrm{m}$ per feature and a color depth of up to 265 grayscale levels applied to a microstructured substrate, the fabrication of such patterned functional surfaces in 3D paves the way for development of cell-instructive surfaces that could regulate a specific cell function. Potential applications of high-definition 
grayscale patterns on thermoformed films include the study of immobilized effectors for chemotaxis in arbitrary shapes or assays for cell differentiation and polarization that take advantage of both the spatial orientation of signals within a 3D artificial niche, as well as a defined relative surface concentration that can be obtained by an MPL-PAP approach. It has been shown that different fluorophores can be immobilized to the surface by irradiation with their respective excitation wavelength ${ }^{[14]}$ so that in the future multiligand patterns could be obtained by using an orthogonal system of different ligands or functional groups coupled to fluorophores with non-overlapping excitation spectra. If necessary the resolution of MPL on polymer films can be further improved in future experiments, alternatively other highly parallelized patterning strategies such as polymer pen lithography might be tested to create high resolution and highly multiplexed functional patterns. ${ }^{[41]}$

To further confirm the flexibility of the SMART technology, we have developed a second module using CVD polymerization. Applications of thermoformed 3D substrates pre-patterned via CVD polymerization include cell-based assays that require a well-defined chemical composition of the surface, as demonstrated here with ATRP-based PEGylation. Vapor-based polymerization can be applied to any substrates since the reactive coating forms a homogeneous polymer layer. ${ }^{[10 a]}$ In addition, a wide variety of functional groups (carbonyl, ester, hydroxyl, amine, alkyne, vinyl, as well as fluorinated groups) may be anchored by this surface modification process due to the availability of numerous [2.2] paracyclophane derivatives. ${ }^{[8]}$ Homogeneous copolymerization, ${ }^{[42]}$ patterning of two or more copolymers, ${ }^{[9]}$ or even creation of continuous functional polymer gradients ${ }^{[33]}$ can be achieved during CVD-coating of the substrate. The patterning strategy, such as microcontact printing, VAMPIR, or Dip-Pen Nanolithography, enables precise definition of the pattern scale-from micro to nano-and its spatial distribution. After thermoforming, the surface functionalities may then be used for further modification, polymerization or immobilization of biomolecules using bio-orthogonal approaches. ${ }^{[44]}$

Noteworthy, our results confirm the applicability of two simple and convenient methods for generation of biofunctionalized patterns on 3D surfaces of thermoformed microstructures. Besides controlling curvature radii, topographies, and por osity, ${ }^{[21 a, 22,23]}$ these novel SMART modules open new avenues for creating biochemically defined microstructures such as artificial niches for 3D cell cultures. ${ }^{[45]}$ The stability of our patterns would also allow their integration into microfluidic systems, for instance, by closing the prepatterned channels via additional thermal bonding step and subsequent orthogonal coupling of biomolecules in a flow-through-mode. Such functionalized 3D microdevices can also be utilized in bioanalytics, including biochemical assays and biosensor systems. The introduced strategies via MPL-PAP and CVD are both suitable to pattern areas within a size range of several $\mathrm{cm}^{2}$ with their maximum resolution.

\section{Conclusions}

We demonstrated two different approaches for biofunctional patterning of $3 \mathrm{D}$ devices for the analysis and control of cell adhesion. With the MPL-PAP method, arbitrary grayscale patterns of ligands with a resolution of $7.5 \mu \mathrm{m}$ could be immobilized on protein-coated surfaces of COP films. Alternatively, using CVD polymerization, chemically-defined binary patterns were created on the surfaces of COP films. Both strategies led to thermostable micropatterns that remained functional after microscale thermoforming and can be decorated with bioactive molecules. These patterned 3D substrates are applicable for the analysis of cell characteristics, such as adhesion, as demonstrated by cultivation of L929 fibroblasts. The unique combination of thermoforming with lithography or vapor-based polymerization allowed engineering of robust functional surfaces and opens new perspectives for a wide range of $3 \mathrm{D}$ in vitro cell culture applications. Due to their flexibility and possible integration of additional effectors for cell behavior, these bioactive patterned 3D cell culture models could be applied for various biological experiments, including regulated differentiation of stem cells.

\section{Experimental Section}

Materials: [2.2]paracyclophane-4-methyl-2-bromoisobutyrate ${ }^{[26]}$ and [2.2]paracyclophane-4-carboxylic acid pentafluorophenolester ${ }^{[10 c]}$ precursors were synthesized as described elsewhere. Poly(ethylene glycol) methyl ether methacrylate (PEGMA, Sigma-Aldrich, Germany) was passed through a neutral alumina column to remove the inhibitor. Unless another supplier is specified, all other chemicals were purchased from Sigma-Aldrich (Germany) and used as received. A commercially available cyclo-olefin polymer film (COP, ZF14-188, Zeon Chemicals Europe) with a thickness of $188 \mu \mathrm{m}$ and a diameter of $40 \mathrm{~mm}$ was used as substrate for patterning and cell culture. For MPL-PAP experiments, sterile petri dishes with a diameter of $35 \mathrm{~mm}$ and $188 \mu \mathrm{m}$ thick COP polymer films as bottom were used (ibidi, Germany).

Surface Patterning by Maskless Projection Lithography: COP polymer films were immersed in $3 \%(\mathrm{w} / \mathrm{v})$ bovine serum albumin (BSA) in phosphate buffered saline (PBS, pH 7.4) for $10 \mathrm{~min}$. For fibronectin coating, the polymer films were incubated in freshly prepared solution

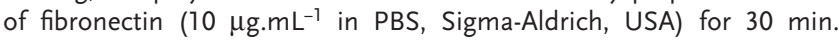
The coating solution was discarded and films were washed three times with PBS for $5 \mathrm{~min}$ under constant shaking, rinsed with water and dried with nitrogen. The patterning step was performed directly after coating. Shortly, a custom-designed MPL system was set-up with a digital micromirror device (DMD, type DLP Discovery 4100, Vialux, Germany) as light modulator as described elsewhere. ${ }^{[15]}$ A customized software enables projection of 8 bit grayscale bitmap-images comprising $1024 \times 768$ pixels. In brief, light from a high-pressure mercury arc lamp (Superlite 400, Lumatec, Germany) is filtered to $490 \mathrm{~nm} \pm 20 \mathrm{~nm}$ using the embedded bandwidth filters. The arc lamp is connected to the projection optics with a flexible light guide. The light is collimated, homogenized and the $3 \mathrm{~mm}$-beam diameter is expanded by appropriate optics to fully and homogeneously illuminate the $17.78 \mathrm{~mm}^{2} \mathrm{DMD}$. Depending on whether a respective mirror of the DMD is in its off- or on-state, light is either reflected to an absorber or towards the sample via demagnification optics, leading to a dark or bright pixel of $2.5 \mu \mathrm{m}$ edge length on the substrate positioned atop the projection optics. Coated films were covered with a solution of fluorescein-5-biotin (F5B, $80 \mu \mathrm{m}$ in PBS) and illuminated from underneath to achieve patterning. Exposure with structured light with a wavelength of $490 \pm 20 \mathrm{~nm}$ generated a primary surface pattern by photobleaching.

Surface Patterning by Chemical Vapor Deposition Polymerization: First, [2.2]paracyclophane-4-methyl-2-bromoisobutyrate was sublimated at $90-110{ }^{\circ} \mathrm{C}$ under reduced pressure $(<0.1 \mathrm{mbar})$ before entering the pyrolysis furnace, maintained at $540{ }^{\circ} \mathrm{C}$, at an argon flow rate of $20 \mathrm{sccm}$. Polymerization of the precursor occurred by vapor-deposition 
on the substrate kept at $15{ }^{\circ} \mathrm{C}$ in the deposition chamber. Then, a patterned PDMS microstencil (squares of $150 \mu \mathrm{m} \times 150 \mu \mathrm{m}$ or $250 \mu \mathrm{m} \times$ $250 \mu \mathrm{m}$ ), fabricated as previously described, ${ }^{\left[{ }^{[9]}\right.}$ was sealed to the coated substrate for the second CVD step. [2.2]Paracyclophane-4-carboxylic acid pentafluorophenolester was sublimated under vacuum, pyrolyzed at $560{ }^{\circ} \mathrm{C}$ and spontaneously polymerized on the cold substrate. Thus, a pattern of poly ( $p$-xylylene-4-carboxylic acid pentafluorophenolester-co-pxylylene) was generated on the surface uncovered by the microstencil.

Microthermoforming of Patterned Films: Microchannels were fabricated by microscale thermoforming on an in-house developed machine with a lockable brass tool. ${ }^{[21 a, 22]}$. An aperture-like brass mold with an outer diameter of $40 \mathrm{~mm}$ and thickness of $300 \mu \mathrm{m}$ was mounted into the lower half of the tool and connected to a vacuum pump for evacuation of the mold cavity from the rear. The patterned film was positioned on the mold in the thermoforming set-up, the pattern being accurately aligned to the central mold cavity with the dimensions $20 \mathrm{~mm} \times 1 \mathrm{~mm} \times$ $0.3 \mathrm{~mm}(\mathrm{I} \times \mathrm{w} \times \mathrm{d})$. After evacuation, the polymer film was heated until it reached a softened state, allowing deformation by applying gas pressure. Upon reaching the forming temperature of $140{ }^{\circ} \mathrm{C}$, a gas pressure of $1 \mathrm{MPa}$ was applied, which resulted in forming of channels with an average depth of $318 \mu \mathrm{m} \pm 8 \mu \mathrm{m}$. Then, the temperature was decreased to $80^{\circ} \mathrm{C}$, the gas pressure was released and the film was further cooled down to $42{ }^{\circ} \mathrm{C}$. Finally, the tool was opened, the COP film was manually demolded and subjected to further surface modifications.

PEGMA Grafting and Biomolecular Immobilization on Patterned CVD Coatings: PEGMA was polymerized by atom transfer radical polymerization (ATRP) from poly(p-xylylene-4-methyl2-bromoisobutyrate-co-p-xylylene) areas on the substrate. The 2-bromoisobutyrate group of the polymer coating acted as the ATRP initiator. An aqueous solution of PEGMA ( 50 wt \%, 200 equiv), $\mathrm{CuBr}$ (1 equiv), $\mathrm{CuBr}_{2}$ ( 0.3 equiv) and 2,2'-bipyridine (bpy, 3 equiv) was degassed via argon bubbling under vigorous stirring for $40 \mathrm{~min}$. Simultaneously, a $50 \mathrm{~mL}$ Erlenmeyer flask, containing a single CVD-coated COP film, was purged and stored under argon. The homogeneous dark-brown solution $(10 \mathrm{~mL})$ was then transferred into the Erlenmeyer flask to completely cover the substrate. The polymerization proceeded at room temperature overnight. After polymerization, the samples were rinsed thoroughly and soaked in distilled water for $4 \mathrm{~h}$. Then, biomolecules were immobilized on the substrate areas coated with poly( $p$-xylylene-4-carboxylic acid pentafluorophenolester-co-p-xylylene). The substrates were incubated with biotin-dPEG ${ }_{7}-\mathrm{NH}_{2} \quad\left(10 \mu \mathrm{g} \mathrm{mL} \mathrm{L}^{-1}\right.$ in phosphate buffered saline (PBS), Celares, Germany) or fibronectin $\left(10 \mu \mathrm{g} \mathrm{mL} \mathrm{m}^{-1}\right.$ in PBS, Sigma Life Sciences, Germany) for $1 \mathrm{~h}$ at room temperature. Substrates were then rinsed with PBS and distilled water. All samples were prepared in duplicates.

Fluorescent Staining of Patterned Films and Imaging: For fluorescent staining of polymer films, a diluted solution of streptavidin-Cy3 or streptavidin-Alexa488 (100 to $200 \mu \mathrm{L}$ of a 1:200 (v:v) dilution in PBS, Life Technologies, USA) was applied to cover the entire patterned area. The solution was incubated in a closed dish for $1 \mathrm{~h}$ in the dark. After removal of the staining solution, the surface was washed with PBS three times for $5 \mathrm{~min}$. The sample was rinsed with distilled water and dried with nitrogen. To determine resolution and saturation of lithography patterns, fluorescent images were recorded with an Axiovert 200M (Carl Zeiss, Germany) inverted fluorescence microscope, equipped with AxioVision software version 4.7 (Carl Zeiss, Germany). Images were exported in TIF-format. The evaluation and image processing were performed in FIJI, ${ }^{[46]}$ a distribution of image. ${ }^{[47]}$

Cell Culture: Mouse L929 fibroblasts (NCTC clone 929, ATCC, USA) were grown in Eagle's Minimal Essential Medium (EMEM, with L-glutamate, ATCC, USA), supplemented with $10 \%$ Fetal Bovine Serum (FBS, ATCC, Germany) and $1 \%$ penicillin/streptomycin (100 x stock, PAA, Austria) at $37{ }^{\circ} \mathrm{C}, 5 \% \mathrm{CO}_{2}$. The cells were cultivated in $75 \mathrm{~cm}^{2}$ flasks (SPL Life Sciences, South Korea) for 1 week until reaching approximately $100 \%$ confluence. The patterned COP films were immersed in distilled water for $1 \mathrm{~h}$, followed by immersion in PBS $+/+$ (containing $\mathrm{Ca}^{2+}$ and $\mathrm{Mg}^{2+}$, PAA, Austria) for $30 \mathrm{~min}$. The $\mathrm{L} 929$ were treated with $0.25 \%$ Trypsin solution (Life Technologies, Germany), counted and 50000 cells were plated per COP substrate, supplied with fresh culture medium 3.5 $\mathrm{mL}$ ) and allowed to adhere for $72 \mathrm{~h}$ at $37^{\circ} \mathrm{C}, 5 \% \mathrm{CO}_{2}$. The $\mathrm{L} 929$ cells were used below passage 33 .

Fluorescence Live-Dead Staining: $72 \mathrm{~h}$ after seeding, the cells were carefully washed in PBS +/+ buffer ( 3 times, $5 \mathrm{~min}$ ) and stained with solutions, containing Calcein-AM (5 $\mu \mathrm{m}$, Sigma-Aldrich, USA) or SYTO16 Green Fluorescent Nucleic Acid Stain (Syto16, $1 \mu \mathrm{m}$, Invitrogen, Germany) and propidium iodide (PI, $500 \mathrm{~nm}$, Sigma Life Sciences, Germany) in EMEM. The cells were incubated for $20 \mathrm{~min}$ at $37^{\circ} \mathrm{C}$ in a $5 \% \mathrm{CO}_{2}$ atmosphere. The staining solution was removed and the samples were rinsed in PBS +/+ buffer ( 3 times, $5 \mathrm{~min}$ ). The combined staining protocols (live-dead staining) with Calcein-AM and PI or Syto 16 and $\mathrm{Pl}$ are used to distinguish the viable cells (green cytosol) from the dead cells (red stained nuclei) by fluorescence microscopy. The edges of the COP films were cut and carefully flipped over on rectangular $0.13 \mathrm{~mm}$ thick sterile glass coverslips $(24 \mathrm{~mm} \times 60 \mathrm{~mm}$, Menzel-Glaeser Germany) and coated with VECTASHIELD mounting medium (25 $\mu \mathrm{L}$, Vector Laboratories, USA) to prevent photobleaching of the samples during imaging and prolonged storage.

\section{Supporting Information}

Supporting Information is available from the Wiley Online Library or from the author.

\section{Acknowledgements}

This work received financial support by the 'Concept for the Future' of Karlsruhe Institute of Technology (KIT) within the framework of the German Excellence Initiative (Research group 26-2 and Young Investigator group 03-109) and Max-Buchner Research fellowship (DECHEMA, MBFSt \#2676) as well as the Biolnterfaces (BIF) program of the KIT. P.M.N. has been supported by DAAD (German Academic Exchange Service) doctoral grant.

Received: March 28, 2013

Revised: May 7, 2013

Published online: July 22, 2013

[1] a) C. Cha, W. B. Liechty, A. Khademhosseini, N. A. Peppas, ACS Nano 2012, 6, 9353; b) P. Zorlutuna, N. Annabi, G. Camci-Unal, M. Nikkhah, J. M. Cha, J. W. Nichol, A. Manbachi, H. Bae, S. Chen, A. Khademhosseini, Adv. Mater. 2012, 24, 1782.

[2] a) J. E. Frith, R. J. Mills, J. J. Cooper-White, J. Cell Sci. 2012, 125, 317; b) S. Musah, S. A. Morin, P. J. Wrighton, D. B. Zwick, S. Jin, L. L. Kiessling, ACS Nano 2012, 6, 10168; c) A. M. Ross, J. Lahann, J. Polym. Sci. B 2013, 51, 775.

[3] a) J. J. Díaz-Mochón, G. Tourniaire, M. Bradley, Chem. Soc. Rev. 2007, 36, 449; b) F. Xu, J. Wu, S. Wang, N. G. Durmus, U. A. Gurkan, U. Demirci, Biofabrication 2011, 3.

[4] R. S. Kane, S. Takayama, E. Ostuni, D. E. Ingber, G. M. Whitesides, Biomaterials 1999, 20, 2363.

[5] E. Delamarche, A. Bernard, H. Schmid, B. Michel, H. Biebuyck, Science 1997, 276, 779.

[6] R. D. Piner, J. Zhu, F. Xu, S. Hong, C. A. Mirkin, Science 1999, 283, 661.

[7] W. He, C. R. Halberstadt, K. E. Gonsalves, Biomaterials 2004, 25, 2055.

[8] H. Y. Chen, J. Lahann, Langmuir 2011, 27, 34.

[9] H. Y. Chen, J. Lahann, Adv. Mater. 2007, 19, 3801.

[10] a) H. Y. Chen, M. Hirtz, X. Deng, T. Laue, H. Fuchs, J. Lahann, J. Am. Chem. Soc. 2010, 132, 18023; b) H. Y. Chen, J. Lahann, Anal. 
Chem. 2005, 77, 6909; c) J. Lahann, I. S. Choi, J. Lee, K. F. Jensen, R. Langer, Angew. Chem. Int. Ed. 2001, 40, 3166.

[11] M. Mayer, J. Yang, I. Gitlin, D. H. Gracias, G. M. Whitesides, Proteomics 2004, 4, 2366.

[12] M. A. Holden, P. S. Cremer, J. Am. Chem. Soc. 2003, 125, 8074.

[13] J. M. Bélisle, J. P. Correia, P. W. Wiseman, T. E. Kennedy, S. Costantino, Lab Chip 2008, 8, 2164.

[14] J. M. Bélisle, D. Kunik, S. Costantino, Lab Chip 2009, 9, 3580.

[15] A. Waldbaur, B. Waterkotte, K. Schmitz, B. E. Rapp, Small 2012, 8, 1570

[16] a) D. E. Discher, P. Janmey, Y. L. Wang, Science 2005, 310, 1139; b) A. M. Ross, Z. Jiang, M. Bastmeyer, J. Lahann, Small 2012, 8, 336.

[17] a) H. Baharvand, S. M. Hashemi, S. K. Ashtiani, A. Farrokhi, Int. J. Dev. Biol. 2006, 50, 645; b) S. Gobaa, S. Hoehnel, M. Roccio, A. Negro, S. Kobel, M. P. Lutolf, Nat. Methods 2011, 8, 949; c) D. Lehnert, B. Wehrle-Haller, C. David, U. Weiland, C. Ballestrem, B. A. Imhof, M. Bastmeyer, J. Cell Sci. 2004, 117, 41.

[18] G. H. Underhill, G. Peter, C. S. Chen, S. N. Bhatia, Annu. Rev. Cell Developmental Biol. 2012, 28, 385.

[19] A. S. Andersson, F. Backhed, A. von Euler, A. Richter-Dahlfors, D. Sutherland, B. Kasemo, Biomaterials 2003, 24, 3427.

[20] a) P. S. Dittrich, A. Manz, Nat. Rev. Drug Discov. 2006, 5, 210; b) P. Neužil, S. Giselbrecht, K. Länge, T. J. Huang, A. Manz, Nat. Rev. Drug Discov. 2012, 11, 620.

[21] a) S. Giselbrecht, T. Gietzelt, E. Gottwald, C. Trautmann, R. Truckenmüller, K. F. Weibezahn, A. Welle, Biomed. Microdevices 2006, 8, 191; b) M. Heilig, S. Giselbrecht, A. Guber, M. Worgull, Microsystem Technol. 2010, 16, 1221; c) R. Truckenmüller, S. Giselbrecht, C. Van Blitterswijk, N. Dambrowsky, E. Gottwald, T. Mappes, A. Rolletschek, V. Saile, C. Trautmann, K. F. Weibezahn, A. Welle, Lab Chip 2008, 8, 1570.

[22] R. Truckenmüller, S. Giselbrecht, N. Rivron, E. Gottwald, V. Saile, A. Van Den Berg, M. Wessling, C. Van Blitterswijk, Adv. Mater. 2011, $23,1311$.

[23] a) S. Giselbrecht, M. Reinhardt, T. Mappes, M. Börner, E. Gottwald, C. Van Blitterswijk, V. Saile, R. Truckenmüller, Adv. Mater. 2011, 23, 4873; b) C. Richter, M. Reinhardt, S. Giselbrecht, D. Leisen, V. Trouillet, R. Truckenmüller, A. Blau, C. Ziegler, A. Welle, Biomed. Microdevices 2010, 12, 787; c) R. Truckenmüller, S. Giselbrecht, M. Escalante-Marun, M. Groenendijk, B. Papenburg, N. Rivron, H. Unadkat, V. Saile, V. Subramaniam, A. Van Den Berg, C. Van Blitterswijk, M. Wessling, J. De Boer, D. Stamatialis, Biomed. Microdevices 2012, 14, 95.

[24] A. M. Greiner, B. Richter, M. Bastmeyer, Macromol. Biosci. 2012, 12, 1301

[25] J. Lahann, Polym. Int. 2006, 55, 1361.

[26] X. Jiang, H. Y. Chen, G. Galvan, M. Yoshida, J. Lahann, Adv. Funct. Mater. 2008, 18, 27.
[27] X. Deng, T. W. Eyster, Y. Elkasabi, J. Lahann, Macromol. Rapid Commun. 2012, 33, 640 .

[28] a) A. P. Marques, R. L. Reis, J. A. Hunt, Biomaterials 2002, 23, 1471; b) M. C. Serrano, R. Pagani, M. Vallet-Regí, J. Peña, A. Rámila, I. Izquierdo, M. T. Portolés, Biomaterials 2004, 25, 5603; c) J. Sun, Z. Dai, Y. Zhao, G. Q. Chen, Biomaterials 2007, 28, 3896.

[29] A. Levesque, A. Paquet, M. Page, Cytometry 1995, 20, 181.

[30] L. Bouchier-Hayes, C. Muñoz-Pinedo, S. Connell, D. R. Green, Methods 2008, 44, 222.

[31] P. Kingshott, S. McArthur, H. Thissen, D. G. Castner, H. J. Griesser, Biomaterials 2002, 23, 4775

[32] a) E. Gottwald, B. Lahni, G. Lüdke, T. Preckel, C. Buhlmann, Biotechniques 2003, 35, 358; b) R. L. Sparrow, E. Tippett, J. Immunol. Methods 2005, 305, 173.

[33] a) M. Jamal, N. Bassik, J. H. Cho, C. L. Randall, D. H. Gracias, Biomaterials 2010, 31, 1683; b) M. Rumpler, A. Woesz, J. W. C. Dunlop, J. T. Van Dongen, P. Fratzl, J. Royal Soc. Int. 2008, 5, 1173.

[34] a) M. Ehrbar, A. Sala, P. Lienemann, A. Ranga, K. Mosiewicz, A. Bittermann, S. C. Rizzi, F. E. Weber, M. P. Lutolf, Biophys. J. 2011, 100, 284; b) A. J. Keung, P. Asuri, S. Kumar, D. V. Schaffer, Integrative Biol. 2012, 4, 1049; c) A. Pathak, S. Kumar, Proc. Natl. Acad. Sci. USA 2012, 109, 10334.

[35] a) D. Huh, G. A. Hamilton, D. E. Ingber, Trends Cell Biol. 2011, 21, 745; b) J. Jiang, E. T. Papoutsakis, Adv. Healthcare Mater. 2013, 2, 25.

[36] a) F. Edalat, I. Sheu, S. Manoucheri, A. Khademhosseini, Curr. Opin. Biotechnol. 2012, 23, 820; b) H. Yu, C. Y. Tay, M. Pal, W. S. Leong, H. Li, H. Li, F. Wen, D. T. Leong, L. P. Tan, Adv. Healthcare Mater. 2013, 2, 442

[37] C. Priesta, Biomicrofluidics 2010, 4, 032206

[38] R. J. Jackman, J. L. Wilbur, G. M. Whitesides, Science 1995, 269, 664.

[39] A. Embrechts, C. L. Feng, C. A. Mills, M. Lee, I. Bredebusch, J. Schnekenburger, W. Domschke, G. J. Vancso, H. Schönherr, Langmuir 2008, 24, 8841.

[40] M. A. Holden, S. Y. Jung, P. S. Cremer, Anal. Chem. 2004, 76, 1838.

[41] F. Brinkmann, M. Hirtz, A. Greiner, M. Weschenfelder, B. Waterkotte, M. Bastmeyer, H. Fuchs, Small 2013, DOI: 10.1002/smll.201203183.

[42] Y. Elkasabi, H. Y. Chen, J. Lahann, Adv. Mater. 2006, 18, 1521.

[43] a) Y. Elkasabi, J. Lahann, Macromol. Rapid Commun. 2009, 30, 57; b) Y. M. Elkasabi, J. Lahann, P. H. Krebsbach, Biomaterials 2011, 32, 1809.

[44] X. Deng, C. Friedmann, J. Lahann, Angew. Chem. Int. Ed. 2011, 50, 6522.

[45] E. Gottwald, B. Lahni, D. Thiele, S. Giselbrecht, A. Welle, K. F. Weibezahn, J. Vis. Exp. 2008, 15, e564.

[46] J. Schindelin, I. Arganda-Carreras, E. Frise, V. Kaynig, M. Longair, T. Pietzsch, S. Preibisch, C. Rueden, S. Saalfeld, B. Schmid, J. Y. Tinevez, D. J. White, V. Hartenstein, K. Eliceiri, P. Tomancak, A. Cardona, Nat. Methods 2012, 9, 676.

[47] C. A. Schneider, W. S. Rasband, K. W. Eliceiri, Nat. Methods 2012, 9, 671. 